\title{
Netrin UNC-6 and the Regulation of Branching and Extension of Motoneuron Axons from the Ventral Nerve Cord of Caenorhabditis elegans
}

\author{
Yoo-Shick Lim, Smita Mallapur, Gautam Kao, Xing-Cong Ren, and William G. Wadsworth \\ Department of Pathology, Robert Wood Johnson Medical School, Piscataway, New Jersey 08854-5635
}

In the Caenorhabditis elegans embryo, some ventral midline motoneurons extend a process circumferentially to the dorsal midline and a process longitudinally along ventral nerve cord interneurons. Circumferential migrations are guided by netrin UNC-6, which repels motoneuron axons dorsally. Although the motoneuron cell bodies and the longitudinal axons are positioned along UNC-6-expressing interneurons in the ventral nerve cord, the circumferential processes extend only from the motoneuron cell bodies and from defined locations along some longitudinal axons. This implies a mechanism regulates motoneuron branching of UNC-6-responsive processes. We show that expression of unc- $6 \Delta \mathrm{C}$, which encodes UNC-6 without domain $\mathrm{C}$, partially rescues circumferential migration defects in unc- 6 null animals. This activity depends on the netrin receptors UNC-5 and UNC-40. These results indicate that UNC-6 $\Delta \mathrm{C}$ can provide the circumferential guidance functions of UNC-6. Furthermore, we show that expression of unc- $6 \Delta \mathrm{C}$ causes motoneuron branching and the extension of processes from abnormal positions along the ventral nerve cord. This activity is also UNC-5- and UNC-40-dependent. We propose that local interactions mediated by domain $\mathrm{C}$ regulate motoneuron branching and responsiveness to the UNC-6 cue.

Key words: netrin; UNC-6; C. elegans; guidance; axon branching; genetics; invertebrate; in vivo
During the development of a nervous system, molecular guidance cues direct the formation of circumferential and longitudinal nerves in a stereotyped spatial and temporal order. Guidance cues have been described that can attract or repel neuronal growth cones, and these cues apparently can act simultaneously to guide an axon migration (Tessier-Lavigne and Goodman, 1996). However, the mechanisms through which axons respond to the various guidance cues to produce an intricate axon scaffold are not well understood. For example, migrations along the dorsoventral axis are guided by cues different from those that direct migrations along the anteroposterior axis. Therefore, axons that must migrate circumferentially and longitudinally to their final destinations apparently use both sets of cues. This is also the case for individual neurons that extend processes along both axes. These observations imply that molecular mechanisms, either intrinsic to the migrating axon or to the local environment, determine whether the axon extends circumferentially or longitudinally.

In Caenorhabditis elegans, circumferential axon migrations are guided by netrin UNC-6 (Hedgecock et al., 1990). Complex spatial and temporal UNC-6 expression patterns are important for pioneering new tracts and for connecting the tracts together in proper order (Wadsworth and Hedgecock, 1996; Wadsworth et al., 1996; Ren et al., 1999). In general, UNC-6 is expressed

\footnotetext{
Received March 10, 1999; revised May 26, 1999; accepted June 2, 1999.

This work was supported by the National Institutes of Health Grant NS33156. We thank Cori Bargmann, Joe Culotti, Edward Hedgecock, Harold Hutter, and Dave Pilgrim for generously providing GFP marker strains, Zeynep Altun-Gultekin, Cori Bargmann, Monica Driscoll, Cheng-Chen Huang, Seonhee Kim, Qun Wang, and Renping Zhou for comments on this manuscript, Christine Mirzayan and Marc Tessier-Lavigne for helpful discussions and sharing their unpublished results, and Rajesh Patel for assistance with electron microscopy.

Correspondence should be addressed to William Wadsworth, Department of Pathology, Robert Wood Johnson Medical School, 675 Hoes Lane, Piscataway, NJ 08854-5635.

Copyright (C) 1999 Society for Neuroscience $\quad 0270-6474 / 99 / 197048-09 \$ 05.00 / 0$
}

ventrally, suggesting that axons are either repelled dorsally or are attracted ventrally (Wadsworth et al., 1996). To reach their final destinations, many UNC-6-responsive axons also migrate longitudinally in one of the two fascicles of the ventral nerve cord. This suggests that growth cone responsiveness to UNC-6 on entering or leaving the ventral nerve cord could be locally regulated. Less is known about the guidance cues required to direct anteroposterior migrations, although genes that regulate longitudinal migrations have been proposed (McIntire et al., 1992; Hekimi and Kershaw, 1993; Wightman et al., 1996; Wolf et al., 1998).

UNC-6 is a laminin-related protein of 591 amino acids (Ishii et al., 1992). Domains comprising residues 1-437 are homologous to the N-terminal domains VI, V-1, V-2, and V-3 of laminin subunits, whereas the domain comprising residues 438-591, designated domain $\mathrm{C}$, has no homology to laminin subunits (Fig. 1). UNC-6 is a member of the phylogenetically conserved netrin family (Serafini et al., 1994; Harris et al., 1996; Mitchell et al., 1996; Lauderdale et al., 1997; Strahle et al., 1997). The vertebrate netrins have been shown to have chemoattractant and chemorepellent activities for developing axons in the embryonic nervous system (Kennedy et al., 1994; Serafini et al., 1994; Colamarino and Tessier-Lavigne, 1995). Similar to the role of UNC-6 in nematodes, vertebrate netrins guide circumferential migrations in the developing spinal cord. Furthermore, in vitro studies using rat metencephalon commissural axons have shown that axons lose responsiveness to vertebrate netrin-1 after crossing ventral midline floor plate cells (Shirasaki et al., 1998), suggesting that the regulation of netrin signaling at the ventral midline is an evolutionarily conserved feature of nervous system development.

We have proposed that UNC-6 is assembled within basement membranes or along cellular surfaces as an extracellular matrix cue and that it interacts with cell surface receptors on migrating axons or cells (Wadsworth and Hedgecock, 1992, 1996). The direction- and tissue-specific guidance activities are mediated by 


\begin{tabular}{|ll} 
Netrin UNC-6 & $\begin{array}{l}\text { Allele } \\
\text { signal peptide } \\
\text { globular }\end{array}$ \\
EGF-like \\
netrin C
\end{tabular}

Figure 1. Modular organization of netrin. Domains VI and V are related to the $\mathrm{N}$ terminus of laminin subunits, whereas domain $\mathrm{C}$ is shared among netrins and is related to the $\mathrm{C}$ terminus of Frzb, an antagonist of the Wnt receptor (Leyns et al., 1997; Wang et al., 1997). The approximate locations of different $u n c-6$ mutations are shown (after Wadsworth et al., 1996). distinct domains of UNC-6 (Hedgecock et al., 1990; Ishii et al., 1992; Wadsworth et al., 1996). Within domain VI, one mutation, ev436, can produce selective defects in mesoblast but not axonal migrations, whereas another allele, ev437, selectively disrupts ventral migrations of both axons and mesoblasts (Fig. 1). Finally, four alleles that selectively cause defects in dorsal migrations each produce a protein that specifically lacks the V-2 epidermal growth factor-like module.

The response to UNC-6 is known to be mediated by the UNC-5 and UNC-40 receptors. UNC-5 is a member of the Ig superfamily and is expressed in motile cells. It is both necessary and sufficient to direct migrations dorsally, away from ventral UNC-6 sources (Leung-Hagesteijn et al., 1992; Hamelin et al., 1993). UNC-40, a C. elegans homolog of the vertebrate protein Deleted in Colorectal Cancer (DCC) and Drosophila Frazzled (Keino-Masu et al., 1996; Kolodziej, 1996), is required in migrating cells that respond to UNC-6 and is also an Ig superfamily member (Chan et al., 1996). Both DCC and the vertebrate homologs of UNC-5 have been implicated as vertebrate netrin receptors (Keino-Masu et al., 1996; Leonardo et al., 1997). As a simple model, UNC-6 is expressed from ventral sources to form gradients. UNC-5 and UNC-40 are expressed in dorsally migrating axons to mediate a repulsive response to UNC-6, whereas UNC-40 and probably other receptors are expressed in ventrally migrating axons to mediate an attractive response to UNC-6.

In this paper, we report biological activities mediated by UNC-6 domain C. The 153 residue $C$ terminus is shared among netrins and is related to the $\mathrm{C}$ terminus of Frzb, an antagonist of the Wnt receptor (Leyns et al., 1997; Wang et al., 1997). To identify activities that require domain $\mathrm{C}$, we expressed in the unc- 6 null animal transgenes that encode the UNC-6 protein without domain C (UNC-6 $\Delta \mathrm{C}$ ). We find that UNC-6 $\Delta \mathrm{C}$ has the cell and axon guidance functions of UNC-6. Even more surprisingly, we also find that UNC- $6 \Delta \mathrm{C}$ causes the ventral midline motoneurons to branch and extend processes circumferentially. This phenotype depends on $u n c-5$ and $u n c-40$. These results suggest that domain $\mathrm{C}$ of UNC- 6 is required to inhibit ventral nerve cord motoneurons from branching and extending circumferentially in response to UNC-6. We propose a model in which domain $\mathrm{C}$ mediates local control of growth cone pathfinding decisions by modifying ligand-receptor interactions at the ventral nerve cord.

\section{MATERIALS AND METHODS}

C. elegans manipulations. C. elegans cultures were maintained by standard methods (Brenner, 1974). For microscopy of living animals, the animals were mounted on a slide in a small drop of M9 buffer on a 5\% agar pad (Sulston and Hodgkin, 1988). The buffer was sometimes supplemented with $25 \%$ ethanol to anesthetize the animals. Transformation was performed as described (Mello et al., 1991; Mello and Fire, 1995) by injecting plasmid construct pIM\#183 with the dominant transformation marker pRF4, which contains rol-6(su1006) (Kramer et al., 1990). The pIM\#183 plasmid was injected at $50 \mathrm{ng} / \mu \mathrm{l}$ for IM145:urIs 77 and at 10 $\mathrm{ng} / \mu \mathrm{l}$ for the other $u n c-6 \Delta \mathrm{C}$ transgenic lines. In some cases (Table 1 ), the plasmid IM\#175 (Ren et al., 1999), a $g f p$ expression vector that uses the unc-119 promoter (Maduro and Pilgrim, 1995) to drive green fluorescent protein (GFP) expression throughout the nervous system, was also coinjected. Strains harboring introduced DNA as an integrated array were constructed by irradiation (Mello and Fire, 1995). Assays were conducted on a sample size of 100 animals from each of at least three independent lines. Behavioral and cellular defects of unc-5, unc-6, and unc-40 mutants were assayed according to the method of Hedgecock et al. (1990). For assaying the extent of dorsal axon migrations (Fig. 2), axons expressing GFP were scored by epifluorescence microscopy for the crossing of three positional references, the center of the ventral muscle quadrant (VSL), the excretory canal (L), and the center of the dorsal muscle quadrant (DSL), each of which is observed by switching to Nomarski differential interference contrast optics.

Molecular techniques. Basic molecular techniques for preparation of plasmid DNA, restriction enzyme digestions, agarose gel electrophoresis of DNA, Western blotting, and other molecular biology methods were by standard methods (Sambrook et al., 1989). Monoclonal antibody 12CA5 (Boehringer Mannheim, Indianapolis, IN) was used to detect UNC-6 $\triangle \mathrm{C}:: \mathrm{HA}$ on Western blots. DD and VD motoneurons were assayed by using polyclonal Anti-GABA antibody (Sigma, St. Louis, MO) as described (McIntire et al., 1992). Plasmid pIM\#183 was constructed by deleting the domain $\mathrm{C}$ coding region, intron 8 through exon 13 sequences (Ishii et al., 1992), from pIM\#97, a plasmid containing genomic unc-6. Transgenic animals containing pIM\#97 sequence express a hemagglutinin (HA) epitope-tagged UNC-6 that is capable of rescuing unc-6 null mutants (Wadsworth et al., 1996). To make the deletion, two fragments that introduce a PstI site were PCR-amplified. The first used the primer pair, 5'-AATTCCGTGATACTTCTCTGCC-3' and 5'GCTGCAGGATACACGGAGTAACTG-3' and pIM\#97 as a template. The second fragment, containing the $3^{\prime}$ untranslated end of unc-6, used the primer pair 5'-GGTAGCGCTGCAGTGAGAAAAACGAAC-3' and T3 primer, with template pIM\#121, a plasmid containing the UNC-6 cDNA sequence. A 4809 bp fragment was exercised with XhoI and PstI from pIM\#97 and replaced with the $1866 \mathrm{bp}$ XhoI-PstI fragment of the first PCR-amplified product. The resulting plasmid was digested with NotI and PstI, and the 552 bp NotI-PstI fragment of the second PCR product was cloned in to generate pIM\#183.

Transgenic animals. The strains used in this paper are IM19, urIs13[IM\#175 pRF4]; IM39, urIs13; unc-6(ev400); IM113, urIs62[IM\#97 IM\#175 pRF4]; unc-6(ev400); IM65, urIs13; unc-5(e53); IM167, urIs13; unc-5(e53); unc-6(ev400); IM62, urIs13; unc-40(e1430); IM98, urIs13; unc-40(e1430); unc-6(ev400); IM94, urIs55[IM\#183 IM\#175 pRF4]; unc-6(ev400); IM114, urIs63[IM\#183 IM\#175 pRF4]; unc-6(ev400); IM201, urIs63; unc-5(e53); unc-6(ev400); IM138, urIs63; unc-40(e1430); unc-6(ev400); IM135, urIs63; unc-104(rh43); unc6(ev400); IM145, urIs77[IM\#183 IM\#175 pRF4]; and IM218, urIs77; unc-6(ev400). Transgenic unc-6 $\Delta \mathrm{C}$ strains were also created that contained GFP markers for different classes of neurons: NW1099, evIs82a 


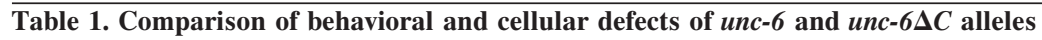

\begin{tabular}{|c|c|c|c|c|c|c|c|c|}
\hline \multirow[b]{2}{*}{ unc- 6 transgene } & \multirow[b]{2}{*}{ Genetic background } & \multicolumn{4}{|c|}{ Dorsal guidance } & \multicolumn{2}{|c|}{ Ventral guidance } & \multirow[b]{2}{*}{$\begin{array}{l}\text { Displaced } \\
\text { axons }^{f}\end{array}$} \\
\hline & & $\mathrm{UNC}^{a}$ & $\begin{array}{l}\text { DTC }^{b} \\
\text { ANT }\end{array}$ & $\begin{array}{l}\text { DTC }^{b} \\
\text { POST }\end{array}$ & $\begin{array}{l}\mathrm{SDQR}^{c} \\
\text { Axon }\end{array}$ & $\mathrm{EGL}^{d}$ & $\begin{array}{l}\mathrm{PVCR}^{e} \\
\text { Axon }\end{array}$ & \\
\hline & Wild type & 0 & 0 & 0 & 3 & 0 & 0 & 0 \\
\hline & $u n c-6(-)$ & 4 & 38 & 62 & 91 & 55 & 39 & 0 \\
\hline & $u n c-5(-)$ & 4 & 40 & 64 & 83 & 0 & 0 & 0 \\
\hline & unc-5(-); unc-6(-) & 4 & 45 & 73 & 95 & 58 & & 0 \\
\hline & unc- $40(-)$ & 2 & 9 & 25 & 57 & 49 & 23 & 0 \\
\hline & unc- $40(-) ;$ unc- $6(-)$ & 4 & 43 & 65 & 94 & 73 & & 0 \\
\hline unc-6(urIs62) $)^{g}$ & $u n c-6(-)$ & 0 & 0 & 0 & 4 & 0 & & 0 \\
\hline unc-6sC(urIs103) & $u n c-6(-)$ & 3 & 2 & 10 & 50 & 25 & 4 & 78 \\
\hline 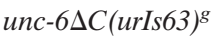 & $u n c-6(-)$ & 3 & 3 & 13 & 56 & 30 & & 80 \\
\hline unc- $6 \Delta C(\text { urIs } 63)^{g}$ & unc-5(-); unc-6(-) & 4 & 40 & 61 & 87 & 25 & & 12 \\
\hline 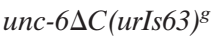 & unc-40(-); unc-6(-) & 4 & 14 & 28 & 65 & 48 & & 30 \\
\hline 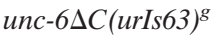 & unc-104(-); unc-6(-) & 4 & & & & & & 30 \\
\hline 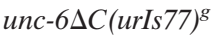 & $u n c-6(-)$ & 3 & 3 & 12 & 38 & 9 & & 75 \\
\hline unc- $6 \Delta C(\text { urIs } 77)^{g}$ & $u n c-6(+)$ & 1 & 6 & 8 & 40 & 0 & & 25 \\
\hline
\end{tabular}

Defects in UNC are ranked from 0 (wild type) to 4 (most severe). For other abnormalities, the percentages of abnormal animals or cells is given.

${ }^{a}$ Uncoordinated movements were ranked by subjective comparison to five reference strains: 0, wild type movement; 4, paralyzed movement (Hedgecock et al., 1990).

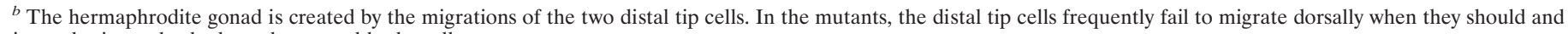
instead migrate back along the ventral body wall.

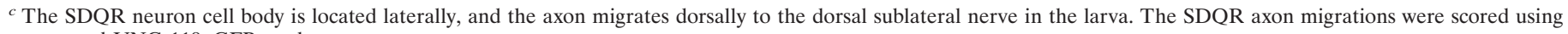
a neuronal UNC-119::GFP marker.

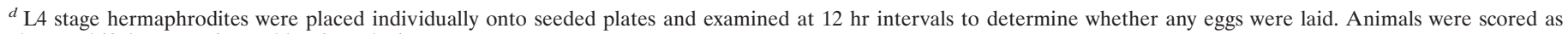
abnormal if they were incapable of egg laying.

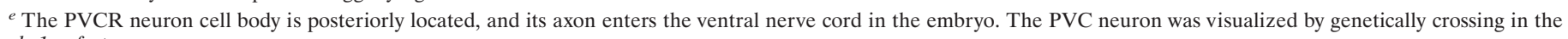
$g l r-1:: .: g f p$ transgene.

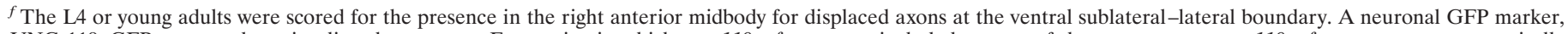

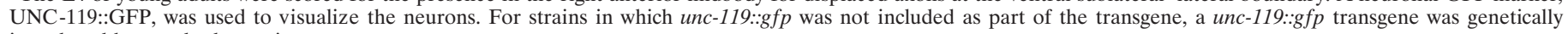
introduced by standard genetic cross.

${ }^{g}$ The transgene includes unc-119:gfp.

[unc-129::GFP pMH86(dpy-20(+))]; IM207, evIs82a; unc-6(ev400); IM210, evIs82a; unc-5(e53); IM211, evIs82a; unc-40(e1430); IM260, evIs82a; unc40(e1430); unc-6(ev400); IM261, evIs82a; unc-5(e53); unc-6(ev400); IM262, urIs103[IM\#183 pRF4]; evIs82a; unc-6(ev400); IM263, urIs103; evIs82a; unc-40(e1430); unc-6(ev400); IM264, urIs103; evIs82a; unc-5(e53); unc6(ev400); IM306, urEx134[unc-129::GFP]; urIs100 [IM\#183 pRF4]; unc6(ev400); and IM307, urEx135[unc-129::GFP]; urIs100; unc-6(ev400), for visualization of DA and DB neurons (Colavita et al., 1998). IM175, rhIs4[glr-1::GFP]; IM202, rhIs4; unc-6(ev400); IM205, rhIs4; unc-5(e53); IM206, rhIs4; unc-40(e1430); and IM258, urIs103; rhIs4; unc-6(ev400), were used for visualization of interneurons axons within the ventral nerve cord (Hart et al., 1995; Maricq et al., 1995). IM259, urIs103; edIs20 [unc-119::GFP]; unc-6(ev400); IM266, urIs100[IM\#183 pRF4]; edIs20; unc-6(ev400); IM267, urIs101[IM\#183 pRF4]; edIs20; unc-6(ev400); IM268, urIs102[IM\#183 pRF4]; edIs20; unc-6(ev400); and IM269, urIs104 [IM\#183 pRF4]; edIs20; unc-6(ev400), were used for visualization of all neurons.

Electron microscopy. Animals were fixed for electron microscopy using glutaraldehyde and then osmium tetroxide (Sulston et al., 1983). Ten L4 stage animals were aligned within a small agar block, embedded, and sectioned together. Serial sections were collected through the anterior midbody region. Sections were poststained with uranyl acetate and lead citrate.

\section{RESULTS}

\section{The expression of unc- $6 \Delta C$ rescues unc- 6 null phenotypes}

To determine whether domain C of UNC-6 is required for netrin guidance activities, animals expressing a transgene that encodes only for the laminin-like domains of the molecule were constructed. We previously had shown that expression of a transgene encoding HA epitope-tagged UNC-6 fully rescues all mutant phenotypes in the unc- 6 null genetic background (Wadsworth et al., 1996). For the experiment described here, we removed the sequence that encodes for domain $\mathrm{C}$ from the unc- $6:: H A$ clone. Five strains that express the UNC-6 protein without domain $\mathrm{C}$ (UNC-6 $-\mathrm{C}$ ) were established by independently integrating the cloned DNA at different chromosomal locations. Because the results were similar for all five lines, the data for the expression of only three of the transgenes are reported (Table 1). The urIs103 transgene differs from the others in that DNA for the expression of a neuronal GFP marker was not co-injected with the unc- $6 \Delta \mathrm{C}$ clone.

We expressed each transgene in unc- 6 null larvae to examine UNC- $6 \Delta \mathrm{C}$ activity. Expression of the UNC- $6 \Delta \mathrm{C}$ protein was confirmed by Western blot analysis, and in each case the transgenic animals exhibit rescue of the $u n c-6(-)$ uncoordinated behavior (Table 1). This indicates that UNC-6 $6 \mathrm{C}$ has UNC-6 guidance activities. To determine in detail the activity that the unc- $6 \Delta \mathrm{C}$ transgene provides, we examined individual migrations that are indicators of the ability of UNC-6 to guide the dorsal and ventral migrations of cells and axons (Hedgecock et al., 1990).

UNC $-6 \Delta C$ guides dorsal axon migrations. In the embryo, axons of the DA and DB neurons migrate circumferentially from ventral cell bodies to form the dorsal nerve cord. We examined these axons using the expression of an unc-129::gfp transgene (Colavita et al., 1998). In unc-6 $\Delta \mathrm{C}$ animals the circumferential processes reach the dorsal midline and form the dorsal nerve cord (Fig. 2; on average five of six axons scored; $n=50)$. In comparison, the axons fail to reach the dorsal midline in unc-6 null larvae (Fig. 2; $n=50)$. We also examined the postembryonic migration of the 
A

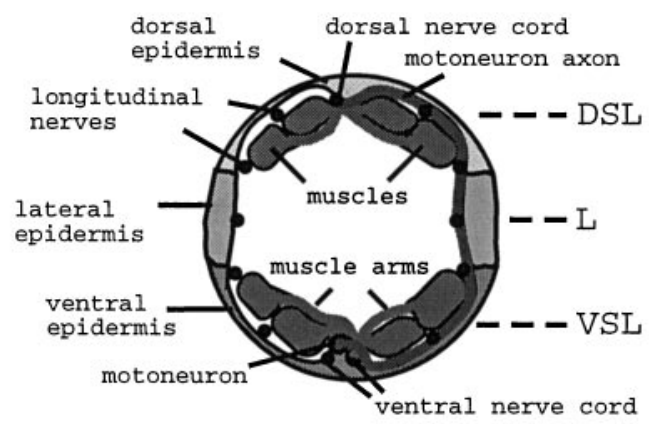

B

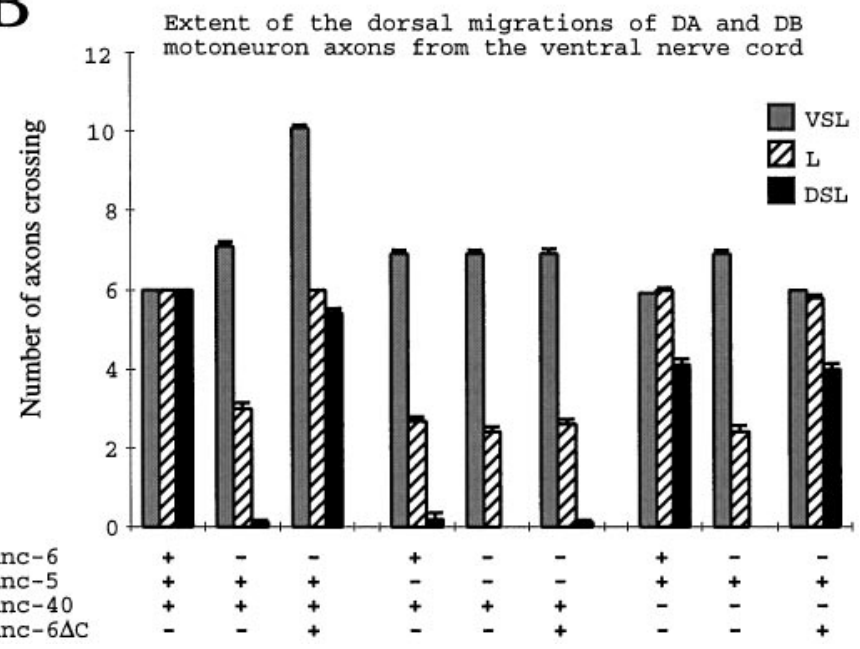

Figure 2. Migrations of the DA and DB motoneuron axons from the ventral nerve cord. $A$, Schematic transverse section of the adult hermaphrodite body wall. The different longitudinal nerves (circles) are located between the basal surface of the epidermis and the basement membranes. Muscle cells project arms to the ventral and dorsal nerve cords to form neuromuscular junctions. The extent of the dorsal migrations of DA and DB motoneuron axons was measured by scoring the number of axons that cross each of three dorsoventral positions: VSL, L, and DSL. In wild-type embryos, processes from DA and DB motoneurons migrate circumferentially from their cell bodies, across all three positions, to the dorsal midline. Each neuron is bipolar, and the second process migrates longitudinally in the ventral nerve cord. B, unc-6, unc-5, and unc-40 are required to guide the axons to the dorsal midline. In the mutants, processes leave the ventral nerve cord and cross the VSL position, but the axons wander and often join longitudinal nerves before reaching the DSL position. Axons also tend to wander in unc-6(-) animals that express the unc- $6 \Delta \mathrm{C}$ transgene, but the same number of axons as in wild-type animals eventually cross the L and DSL positions to reach the dorsal midline, and a dorsal nerve cord is formed. Also, additional processes leave the ventral nerve cord and cross the VSL position. These axons fail to migrate across the lateral epidermis to position L. In unc-5; unc-6 and unc-40; unc-6 mutants that express unc- $6 \Delta \mathrm{C}$, the number of axons that leave the nerve cord and cross the VSL position is near normal, but fewer axons reach the DSL position because the axons wander. Axons were scored on both sides of the animal, but only circumferential migrations on the left side, between the retrovesicular ganglion (near the terminal bulb of the pharynx) and the vulva, are reported here (DA1, DB2, DA3, DB4, DA4, DB5 motoneuron axons). Data points are means $\pm \operatorname{SEM}(n=50)$.

SDQR axon, which migrates dorsally and then anteriorly along the dorsal sublateral nerve. In unc- 6 null larvae, $91 \%$ of the axons fail to migrate dorsally, whereas in unc- 6 null larvae that express the unc- $6 \Delta \mathrm{C}$ transgene, $56 \%$ fail to migrate dorsally $(n=100$ for each; Table 1). Our results indicate that expression of the unc$6 \Delta \mathrm{C}$ transgene can guide dorsal axon migrations.

As a measure of the ability of UNC- $6 \Delta \mathrm{C}$ to guide dorsal cell migrations, we examined distal tip cell migrations. During formation of the hermaphrodite gonad, these two cells migrate longitudinally, one posteriorly and the other anteriorly, along the ventral midline. Each cell then turns and migrates circumferentially to the dorsal midline. In unc- 6 hermaphrodites, the cells frequently fail to migrate dorsally and instead turn back and travel along the ventral surface of the body wall (Hedgecock et al., 1990). Whereas $38 \%$ of anterior distal tip cells fail to migrate dorsally in unc-6 null larvae, $3 \%$ fail in unc- 6 null larvae that express the unc- $6 \Delta \mathrm{C}$ transgene $(n=100$ for each; Table 1$)$. For the posterior distal tip cell, $62 \%$ fail to migrate dorsally in unc- 6 null larvae, and $13 \%$ fail in unc- 6 null larvae that express the unc- $6 \Delta \mathrm{C}$ transgene ( $n=100$ for each; Table 1$)$. These results indicate that expression of the unc- $6 \Delta \mathrm{C}$ transgene can partly rescue the dorsal cell migrations defects of unc- 6 null mutants.

We next examined whether the unc-6 $6 \mathrm{C}$ transgene rescues ventral cell and axon migrations. In hermaphrodites, the anchor cell moves to the center of the ventral uterus and induces ventral epidermal cells to become vulval precursors cells (Sternberg and Horvitz, 1986). In unc-6 mutants, the anchor cell can fail to migrate to the correct ventral position and is therefore displaced laterally or dorsally. Because the vulva does not properly form in this case, the animals are unable to lay eggs as adults (Hedgecock et al., 1990). Although 55\% of unc-6 larva are incapable of egg laying, $30 \%$ of the unc- 6 null larvae that express the unc- $6 \Delta \mathrm{C}$ transgene are incapable of egg laying ( $n=100$ for each; Table 1$)$.

Finally, we examined the ventral axon migration of the PVCR using expression of a $g l r-1:: g f p$ transgene (Hart et al., 1995; Maricq et al., 1995). Compared with $39 \%$ of PVCR axons that fail to migrate to the ventral nerve cord in unc- 6 null larvae, $4 \%$ fail in $u n c-6$ null larvae that express the $u n c-6 \Delta \mathrm{C}$ transgene $(n=100$ for each; Table 1). These results indicate that expression of the unc- $6 \Delta \mathrm{C}$ transgene can partly rescue the ventral migration defects.

\section{The expression of unc-6 $\mathbf{C}$ induces branching and the extension of processes from the ventral nerve cord motoneurons}

We observed the pattern of axon migrations in $u n c-6 \Delta \mathrm{C}$ animals using unc-119:: gfp expression to visualize all neurons. Expression of the unc- $6 \Delta \mathrm{C}$ transgene causes an extraordinary phenotype that we have not observed in $u n c-6(-)$ animals or in animals ectopically expressing unc-6(+) transgenes (Ren et al., 1999). From the ventral nerve cord, axons migrate dorsally across the ventral sublateral surface of the body wall to the boundary of the ventral sublateral and lateral epidermis (Fig. 3). Here the axons either turn longitudinally and travel along the boundary or they terminate abruptly (Fig. $3 B$ ). We scored this phenotype on the right side of the body wall between the vulva and the retrovesicular ganglion (which lies near the terminal bulb of the pharynx). In wild-type animals, no axons migrate to this boundary in the region; however in $80 \%$ of the unc- $6 \Delta \mathrm{C}$ animals axons migrate from the ventral cord to the boundary (Table $1 ; n=100$ ).

We determined that the axons that migrate from the ventral nerve cord to the ventral sublateral-lateral boundary in unc- $6 \Delta \mathrm{C}$ animals are motoneuron axons. The DA and DB motoneurons each extend two processes during embryogenesis (see Fig. 6). The first process extends circumferentially from the ventral midline cell body, and the second process migrates longitudinally as part of the developing ventral nerve cord. In $u n c-6 \Delta \mathrm{C}$ animals, processes migrate dorsally not only from the cell bodies but also from the right ventral nerve cord fascicle. The latter processes migrate 

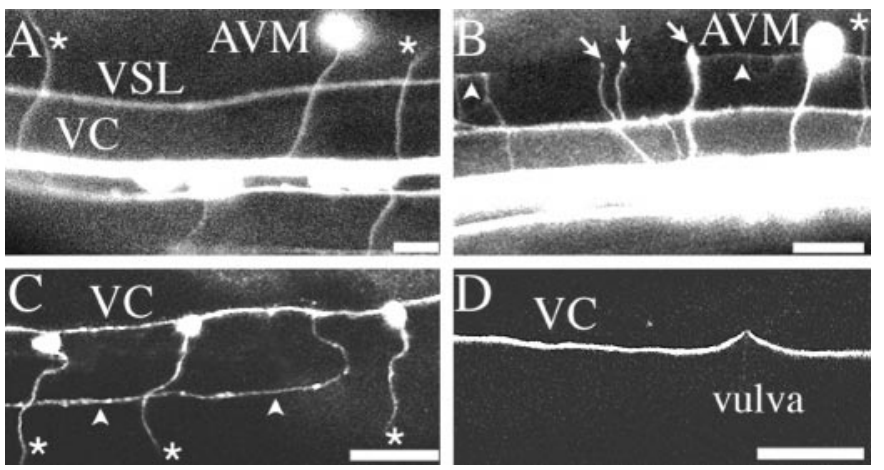

Figure 3. Circumferential axon migrations on the ventral epidermis. $A$, $B$, GFP expression throughout the nervous system. $C$, GFP expression in the DA and DB motoneurons. $D$, GFP expression in ventral nerve cord interneurons. $A$, In wild-type animals, the major ventral longitudinal nerves are the ventral sublateral nerve $(V S L)$ and the ventral nerve cord $(V C)$. The ventral nerve cord is asymmetrical with more axons in the right fascicle than the left. The cell bodies of the ventral motoneurons are positioned along the ventral midline, between the ventral nerve cord fascicles. Circumferential migrations include motoneuron axons (asterisks) that leave the nerve and migrate dorsally to form the dorsal nerve cord (out of the plane of view) and AVM axons that migrate ventrally to the ventral nerve cord. $B$, Expression of the unc- $6 \Delta \mathrm{C}$ transgene induces axons from the ventral nerve cord to migrate dorsally to the ventral sublateral-lateral boundary (arrowheads). Some axons abruptly terminate at the ventral sublateral-lateral boundary (arrows). As in wild-type animals, AVM axons migrate to the ventral nerve cord, and motoneuron axons migrate to the dorsal midline. $C$, In unc-6 $6 \mathrm{C}$ transgenic animals, DA and DB motoneuron axons leave the cell body and migrate to the dorsal midline (asterisks). However, additional processes extend from the right ventral nerve cord fascicle to either the right or left ventral sublateral-lateral boundary (arrowheads). $D$, In unc-6 $6 \mathrm{C}$ transgenic animals, interneuron migrations along the right fascicle of the ventral nerve cord are normal. All micrographs show a dorsal aspect of the ventral midline along the right anterior midbody. Anterior is to the left. Scale bars: $A, B$, $10 \mu \mathrm{m} ; C, D, 50 \mu \mathrm{m}$. Confocal imaging was performed on live animals using extended depth of focusing, which distorts the actual distances between longitudinal tracts because the animal is cylindrical.

on either side of the animal to the ventral sublateral-lateral boundary (Fig. 3C). Because the D class of motoneurons pioneer circumferential tracts, we examined the pattern of the axon migrations of six DA and DB neurons. The numbers of axons that cross over the ventral sublateral, lateral, and dorsal sublateral positions on the left side of the animal between the vulva and the retrovesicular ganglion were scored. As a result of the additional circumferential migrations from the ventral nerve cord, an average of 10 axons migrate over the ventral sublateral position in unc- $6(-)$ animals that express the unc- $6 \Delta \mathrm{C}$ transgene. This is compared with an average of six axons that cross over the position in wild-type animals and 7 in $u n c-6(-)$ animals (Fig. $2 ; n=50$ ). The slightly higher score in $u n c-6(-)$ animals results from wandering axons recrossing the position. Because the processes that leave from the fascicle rarely migrate further than the ventral sublateral-lateral boundary, the average number of axons that cross the lateral and dorsal lateral positions in $u n c-6 \Delta \mathrm{C}$ animals is 6 , the same as in wild-type animals (Fig. $2 ; n=50$ ).

We also examined DD and VD motoneurons using anti-GABA antibodies (Desai et al., 1988; McIntire et al., 1992). Each of these motoneurons sends a process longitudinally at the ventral nerve cord, and from this process a branch extends circumferentially to the dorsal nerve cord. VD motoneurons are born postembryonically. Like the DA and DB motoneurons, additional processes are observed along the ventral sublateral epidermis in unc- $6 \Delta \mathrm{C}$ ani-
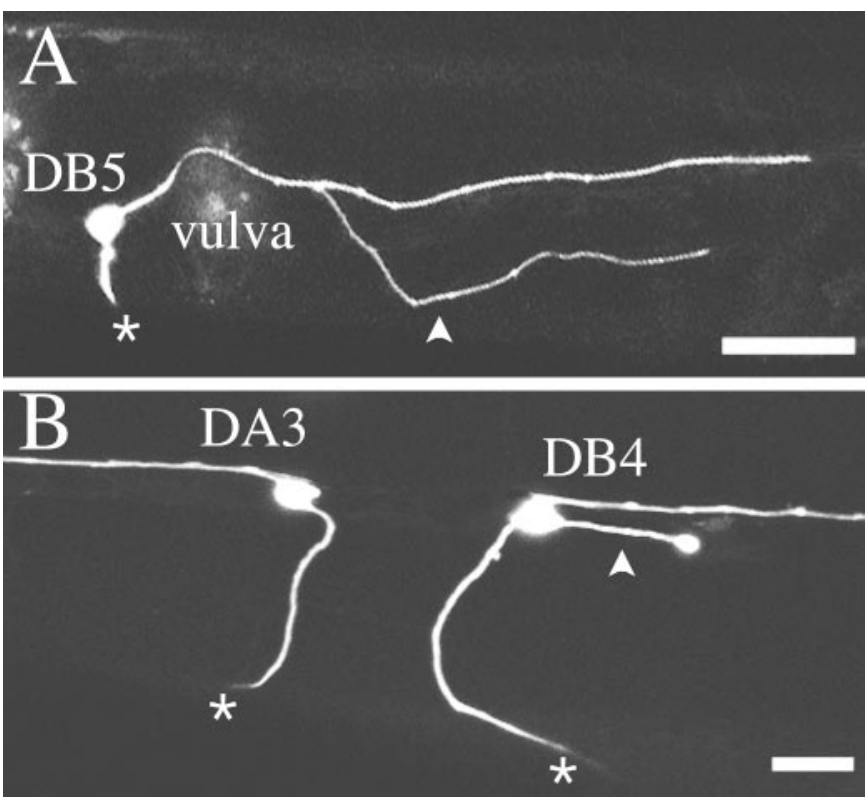

Figure 4. DA and DB motoneurons in unc- $6 \Delta \mathrm{C}$ transgenic animals. Individual motoneurons were visualized by mosaic GFP expression in DA and DB motoneurons. A, Branching of a DB5 motoneuron axon. A normal circumferential axon (asterisk) extends to the dorsal nerve cord (out of the plane of view). From the longitudinal process in the right fascicle of the ventral nerve cord, an addition process extends to the ventral sublateral-lateral boundary (arrowhead). $B$, Ectopic process of a DB4 motoneuron. Although the DA3 motoneuron is normal, having one circumferential process (asterisk) and a longitudinal process that extends anteriorly along the ventral nerve cord, the DB4 motoneuron has an ectopic process extending from the cell body (arrowhead) in addition to its normal circumferential process and posteriorly directed longitudinal process. Scale bars, $25 \mu \mathrm{m}$.

mals. The boundary phenotype in the right anterior region is observed in $83 \%$ of $u n c-6 \Delta \mathrm{C}$ animals $(n=29)$.

In the previous experiments, the processes that leave the ventral nerve cord between the motoneuron cell bodies suggest that the longitudinal axons branch in the unc6- $\Delta \mathrm{C}$ animals. However, we could not reliably distinguish individual processes of the motoneurons because all the motoneurons are GFP-tagged, and the processes overlap in the ventral cord. To determine individual trajectories, we examined animals with mosaic expression of the unc-129::gfp transgene. In rare cases $(<1$ in 100 animals in our strains), the extrachromosomal transgene marker is lost in a subset of DA and DB motoneurons, and the neighboring motoneurons, which retain the transgene, can be individually viewed by epifluorescence microscopy. We observe that the longitudinal motoneuron axons branch and extend processes (Fig. 4A). Furthermore, we observe that ectopic processes also branch from the motoneuron cell bodies (Fig. 4B). These results confirm that expression of unc- $6 \Delta \mathrm{C}$ induces branching of the ventral nerve cord motoneurons.

Unlike the longitudinal motoneuron axons, the interneuron axons along the ventral midline are normal in unc- $6 \Delta \mathrm{C}$ animals. Using expression of a $g l r-1:: g f p$ transgene, 16 interneuron axons (AVAL, AVAR, AVBL, AVBR, AVDL, AVDR, AVEL, AVER, AVG, AVJL, AVJR, DVC, PVCL, PVCR, PVQL, and PVQR) that migrate longitudinally along the ventral nerve cord were examined (Hart et al., 1995; Maricq et al., 1995). None of the axons abnormally leaves the ventral nerve cord in $u n c-6 \Delta \mathrm{C}$ animals (Fig. $3 D ; n=50$ ). We conclude that unc- $6 \Delta \mathrm{C}$ expression only affects the motoneurons in the ventral nerve cord. 


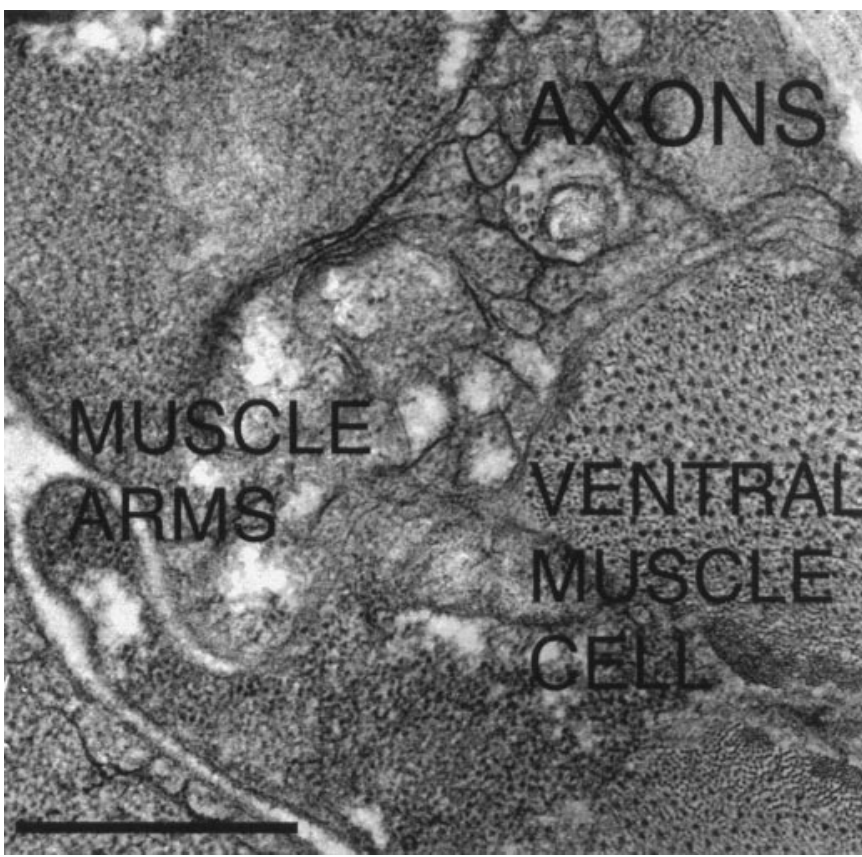

Figure 5. Electron micrograph of motoneuron axons at the ventral sublateral-lateral boundary in an unc- $6 \Delta \mathrm{C}$ transgenic adult hermaphrodite sectioned transversally at the anterior midbody region. The axons are positioned dorsal to the ventral body muscle cells. Arms from ventral body wall muscle cells interdigitate with each other and press against the axons. In wild-type animals, arms from ventral body muscle cells project only to the ventral nerve cord. Scale bar, $0.5 \mu \mathrm{m}$.

We next examined whether the motoneuron axons at the ventral sublateral-lateral boundary in $u n c-6 \Delta \mathrm{C}$ animals retain properties associated with motoneurons of the ventral nerve cord. In C. elegans, ventral muscle cells project an arm to the ventral nerve cord where they form neuromuscular junctions with ventral nerve cord axons. In larvae expressing the unc- $6 \Delta \mathrm{C}$ transgene, the ventral muscle cells extend arms to the ventral sublateral-lateral boundary where they form neuromuscular junctions with the axons (Fig. 5). It is hypothesized that motoneuron axons of the ventral cord secrete a substance that attracts the arms from nearby muscles and that this attractant is translocated via synaptic vesicles along axonal microtubules using kinesin UNC-104 (Hall and Hedgecock, 1991; Otsuka et al., 1991). We observe that the boundary phenotype is partly suppressed by unc-104(rh43). Whereas $80 \%$ of the unc- 6 animals that express unc- $6 \Delta \mathrm{C}$ have axons at the boundary, $30 \%$ of unc-104; unc- 6 animals that express $u n c-6 \Delta \mathrm{C}$ have the phenotype (Table $1 ; n=100$ ). These results indicate that the axons at the ventral sublateral-lateral boundary retain properties associated with motoneuron axons of the ventral nerve cord. The results also suggest that the ability to form neuromuscular junctions between the ventral muscles and the axons at this position may be one reason why the axons often turn longitudinally at the boundary.

\section{unc-5 and unc-40 mediate responses to UNC-6 $\Delta \mathrm{C}$}

To determine the roles that the UNC-6 receptors UNC-5 and UNC-40 play in mediating the UNC-6 $\Delta \mathrm{C}$ signal, we constructed unc-5; unc-6 and unc-40; unc-6 mutants that express the unc- $6 \Delta \mathrm{C}$ transgene. As previously shown for unc-6 (Hedgecock et al., 1990), unc-5 or unc-40 affects the ability of unc-6 $6 \mathrm{C}$ to guide circumferential migrations (Table 1). Moreover, in unc-5 and unc-40 mutants the number of axons that leave the ventral cord is suppressed compared with unc-6 $\mathrm{CC}$ animals. Although $80 \%$ of the unc- 6 null larvae that express the unc- $6 \Delta \mathrm{C}$ transgene have axons at the boundary, $12 \%$ of the unc-5; unc- 6 and $30 \%$ of the unc-40; unc-6 larvae that express the unc- $6 \Delta \mathrm{C}$ transgene have the phenotype (Table $1 ; n=100$ ). For the axon migrations of the six DA and DB motoneurons, on average seven axons cross the ventral sublateral position in unc-5; unc-6 animals, and six cross in unc-40; unc-6 animals that express the unc- $6 \Delta \mathrm{C}$ transgene (Fig. 2; $n=50)$. In comparison, 10 axons cross the ventral sublateral position in unc- 6 animals that express the unc- $6 \Delta \mathrm{C}$ transgene. Finally, whereas an average of five axons reach the dorsal sublateral position in $u n c-6(-)$ animals that express the $u n c-6 \Delta \mathrm{C}$ transgene, rarely do the DA or DB axons reach this position in unc-5; unc- 6 animals or unc-40; unc- 6 animals that express the $u n c-6 \Delta \mathrm{C}$ transgene. These results indicate that the axonal responses to UNC- $6 \Delta \mathrm{C}$, like the responses to UNC-6, are mediated by the UNC-5 and UNC-40 receptors.

\section{UNC-6 $\mathbf{C}$ competes with UNC-6 in vivo}

Expression of most unc- $6 \Delta \mathrm{C}$ transgenes in the wild-type unc$6(+)$ background does not cause mutant phenotypes, suggesting that the endogenous UNC-6 suppresses UNC-6 $\Delta \mathrm{C}$ activity. Like other $u n c-6 \Delta \mathrm{C}$ transgenes, the urIs 77 transgene rescues unc- 6 null phenotypes and induces branching and the migration of processes from the ventral nerve cord (Table 1). However, in the unc-6(+) background, expression of urIs 77 causes a weak uncoordinated (unc) phenotype and mild disruption of circumferential migrations (Table 1). Moreover, the ventral sublateral-lateral boundary phenotype is present in $25 \%$ of the animals along the right anterior body wall. We infer that in urIs 77; unc-6(+) animals the levels of UNC-6 $\Delta \mathrm{C}$ are high enough to dominantly interfere with the activity of endogenous UNC-6. Together, these observations indicate that UNC- $6 \Delta \mathrm{C}$ has a unique activity in the ventral nerve cord that is antagonistic to UNC-6.

\section{DISCUSSION}

\section{The structural domains of UNC-6 mediate distinct biological activities}

We have shown that the expression of $u n c-6 \Delta \mathrm{C}$ rescues circumferential migration defects of unc- 6 null animals, demonstrating that the laminin-related domains of UNC- 6 mediate netrin guidance activities in vivo. The laminin-related structural domains of UNC-6 are associated with both direction- and tissue-specific guidance functions (Wadsworth et al., 1996). These domains are conserved structures found in extracellular matrix proteins, and they mediate interactions between extracellular matrix proteins and between matrix proteins and cellular receptors (Engel, 1996). Although domain $\mathrm{C}$ is not required, it is likely important for the circumferential guidance activity of UNC-6 in vivo, because the expression of the unc- $6 \Delta \mathrm{C}$ transgenes does not rescue the unc- 6 null phenotypes as well as the expression of $u n c-6(+)$ transgenes. Dorsal and ventral UNC-6 guidance functions are similarly affected by unc- $6 \Delta \mathrm{C}$ expression. Our results indicate that domain $\mathrm{C}$ regulates how specific axons respond to UNC-6 in vivo. Domain $\mathrm{C}$ is known to be the major heparin-binding domain, and it is not required by netrin-1 for promoting commissural axon outgrowth from rat spinal cord explants or for binding to DCC, the vertebrate homolog of UNC-40 (Keino-Masu et al., 1996; C. Mirzayan and M. Tessier-Lavigne, personal communication).

\section{Axon migrations of ventral nerve cord motoneurons}

The DA, DB, and DD motoneuron cell bodies are arranged in a row along the ventral midline. In the mature animal, the two 

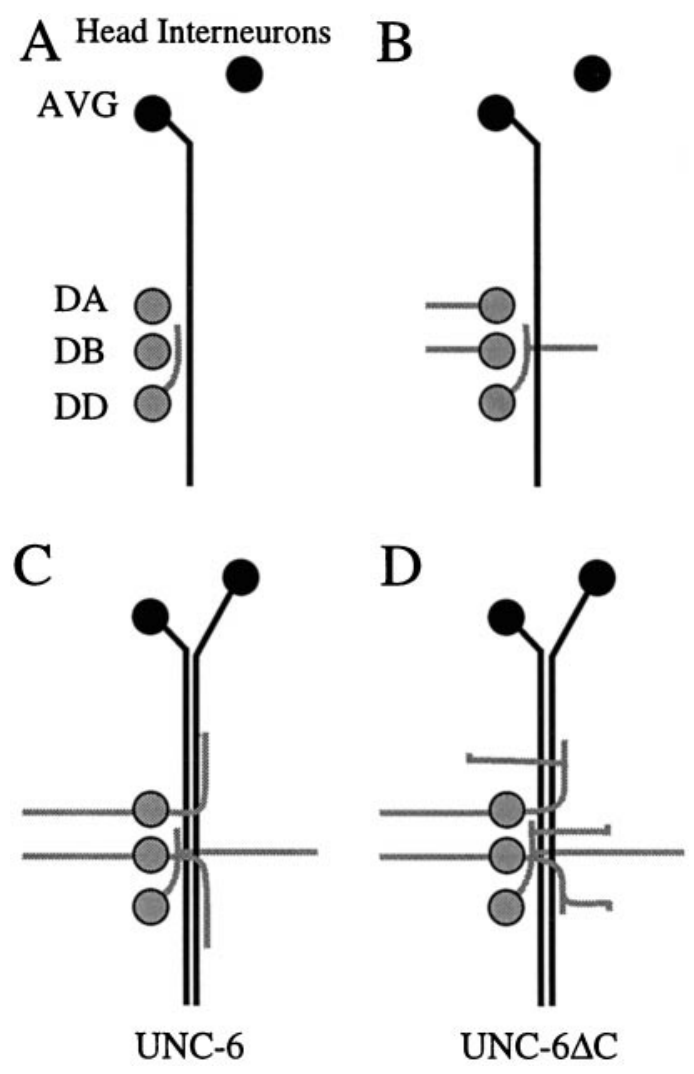

Figure 6. Behavior of DA, DB, and DD motoneuron axons during early development of the ventral nerve cord. $A-D$, Schematic diagrams showing the outgrowth of DA, DB, and DD motoneuron axons in the embryo (after Durbin, 1987). The motoneurons (9 DA, $7 \mathrm{DB}$, and 6 DD) are arranged in a row at the ventral midline, and fascicles of the ventral nerve cord first develop on the right side and then on the left side of the cell bodies. UNC-6 is expressed before axon outgrowth by the underlying ventral epidermal cells and then during outgrowth by the interneurons AVG, AVA, and AVB (Wadsworth et al., 1996). The body wall is shown opened along the dorsal midline in cylindrical projection. D motoneurons are in gray, and UNC-6-expressing interneurons are in black. Anterior is at top. $A$, Process from the DD motoneuron extends anteriorly along the AVG axon, which has pioneered the right side of the ventral nerve cord. $B$, Simultaneously, processes extend dorsally from all three classes of motoneurons. The DA and DB processes extend from the cell bodies, whereas the DD processes always extend from positions opposite the DB cell bodies. $C$, Additional interneurons enter the ventral nerve and the DA and DB motoneurons extend a second process longitudinally along the ventral nerve cord. The earlier DA and DB turn longitudinally along the left side of the dorsal midline to form the dorsal nerve cord (not depicted). $D$, In unc- $6 \Delta \mathrm{C}$ animals, the motoneuron processes that normally extend only longitudinally in the ventral nerve cord instead leave the fascicle extend dorsally.

fascicles of the ventral nerve cord flank the motoneuron cell bodies. The early development of the ventral nerve cord and the outgrowth of the motoneuron processes have been described from electron microscope serial reconstructions (Durbin, 1987). First, the AVG axon migrates posteriorly from an anterior midline cell body and pioneers the right side of the ventral cord. This is followed by DD processes extending forward along the AVG axon (Fig. 6A). Soon after, processes from all three classes of motoneurons simultaneously migrate dorsally (Fig. $6 B$ ). The processes of DA and DB motoneurons extend from the cell bodies, whereas the processes from DD motoneurons extend from near the anterior ends of their axons. Interestingly, the DD motoneuron circumferential extensions are always directly opposite DB cell bodies, suggesting that proximity to the DB cell body influences the point of outgrowth. After the motoneuron processes reach the dorsal midline and begin to form the dorsal nerve cord, additional interneuron axons from cell bodies in the head migrate along the ventral nerve cord. At the same time, from each DA and DB cell body a second process grows out along the ventral nerve cord (Fig. 6C).

\section{A model for the role of domain $\mathbf{C}$ and the direction of motoneuron axon migrations}

Several guidance cues must work in concert to direct motoneuron axon migrations. Interestingly, the ventral nerve cord interneurons express several guidance cues that the motoneuron processes are responsive to. Fasciculation cues of the AVG pioneer and the later interneurons that innervate $\mathrm{DA}$ and $\mathrm{DB}$ to complete the motor circuitry are predicted to attract and guide the migrations longitudinally in the developing nerve cord (Fig. 6). In addition, AVG and the later interneurons also express UNC-6 (Wadsworth et al., 1996).

Domain $\mathrm{C}$ determines whether motoneuron processes migrate longitudinally along the interneurons in the ventral nerve cord or whether they branch and extend processes circumferentially in response to UNC-6. In wild-type animals, UNC-6-responsive processes branch and extend dorsally only at the motoneuron cell bodies and at defined positions along some of the longitudinal processes. Because the motoneurons are positioned along UNC6-expressing interneurons, some mechanism must regulate where the branching occurs. We find that in $u n c-6 \Delta \mathrm{C}$ animals, extra processes extend from the ventral nerve cord and are guided dorsally by UNC- $6 \Delta \mathrm{C}$. A simple model is that domain $\mathrm{C}$ mediates an activity at the ventral midline that prevents motoneuron processes from branching and responding to UNC-6. During the period of pioneer circumferential migrations in wild-type embryos, branching could be induced at the DA and DB motoneurons by molecules that block this activity.

We propose that multimeric receptor complexes on the surfaces of the interneurons and motoneuron axons control the balance between axon-axon adhesion and repulsion by UNC-6. Our results suggest that UNC-5 and UNC-40 are components of these complexes, because $u n c-5$ and $u n c-40$ null mutations can suppress the branching response of the motoneurons to UNC$6 \Delta$ C. Similar to what has been proposed for FGFR activation (Spivak-Kroizman et al., 1994; Walz et al., 1997), oligomerization of UNC- 6 by the binding of domain $\mathrm{C}$ to interneuron-associated proteoglycans could help aggregate and activate the receptor complexes. Besides promoting axon-axon adhesion, these complexes could prevent UNC-5 and UNC-40 from mediating a repulsive response to UNC-6. In contrast, UNC-6 $\Delta \mathrm{C}$, which could not promote receptor aggregation, could interact with UNC-5 and UNC-40 and elicit repulsive and branching signals.

If domain $\mathrm{C}$ helps tether UNC-6 at interneuron surfaces, our model predicts that UNC- $6 \Delta \mathrm{C}$ might diffuse further from the ventral nerve cord. However, this difference by itself does not account for the ventral cord phenotypes of unc- $6 \Delta \mathrm{C}$ animals. First, the diffusion of UNC- $6 \Delta \mathrm{C}$ would be predicted to cause the peak of the repellent gradient to be reduced at the interneurons, and this should favor migration along the interneurons rather than repulsion from them. Second, other experiments show that altering the distribution of UNC-6 does not induce branching and the extension of additional processes from the ventral nerve. For example, these phenotypes are not observed in unc-6 null mutants 
or in animals in which UNC-6 is ectopically expressed either from the touch receptor neurons or from neurons throughout the nervous system (Ren et al., 1999). Furthermore, the distribution of UNC- $6 \Delta \mathrm{C}$ is probably not entirely different from that of UNC-6 in wild-type animals, because circumferential migrations are guided by the expression of $u n c-6 \Delta \mathrm{C}$. Also, in any given unc- $6 \Delta \mathrm{C}$ animal, the longitudinal motoneuron processes will migrate dorsally without preference for one side of the animal (Fig. 3 ), suggesting that the peak of the repellent gradient has not been shifted to one side of the ventral cord. The normal circumferential processes keep their preference in unc-6 $6 \mathrm{C}$ animals. Together these observations suggest that the instructive properties of the UNC- $6 \Delta$ C gradient are similar to those of the UNC- 6 gradient in wild-type animals.

The motoneuron processes that have been induced to leave the ventral nerve cord by $u n c-6 \Delta \mathrm{C}$ expression migrate dorsally along either side of the body wall. At the boundary between the ventral sublateral and lateral body wall, these motoneuron axons either turn or they terminate. This suggests that inhibitory molecules associated with the lateral epidermis surface or with the lateral basement membrane prevent migration across the lateral epidermis. The ventral sublateral-lateral boundary is demarcated by the junction of ventral and lateral epidermal cells. In addition to encountering a different cellular substrate, the axons also encounter distinct basement membranes (Graham et al., 1997; C.-C. Huang, G. Kao, and W. G. Wadsworth, unpublished results). We speculate that the unique appearance and trajectories of these axons are because they are simultaneously directed dorsally by UNC- $6 \Delta C$, although being inhibited from migrating across the lateral epidermis by cues associated with the lateral body wall. This further suggests that the motoneuron axons that normally migrate circumferentially have specific properties that allow them to migrate to the dorsal midline. These properties may only be conferred on the axons that extend during the normal period of circumferential migrations and not on axons that extend in response to UNC-6 $6 \mathrm{C}$ at other times.

$\mathrm{UNC}-6 \Delta \mathrm{C}$ acts as an antagonist to the action of domain $\mathrm{C}$ at the ventral nerve cord. In wild-type animals, the ventral cord phenotype can be induced by the expression of the urIs 77 transgene, suggesting that UNC-6 $\Delta \mathrm{C}$ dominantly interferes with endogenous UNC-6. Furthermore, recent genetic screens have isolated suppressors of the $u n c-6 \Delta \mathrm{C}$ ventral cord phenotype that do not effect $u n c-6$ guidance activities, indicating that this new activity is genetically separable from UNC-6 circumferential guidance activities (Q. Wang and W. G. Wadsworth, unpublished data). Finally, our results provide evidence that in vivo the influence of guidance cues can be modified locally to alter axon trajectories. By regulating spatial and temporal expression patterns and by locally modifying activities, a few guidance cues could direct the development of complex axon scaffolds.

\section{REFERENCES}

Brenner S (1974) The genetics of Caenorhabditis elegans. Genetics 77:71-94.

Chan SS, Zheng H, Su MW, Wilk R, Killeen MT, Hedgecock EM, Culotti JG (1996) UNC-40, a C. elegans homolog of DCC (Deleted in Colorectal Cancer), is required in motile cells responding to UNC-6 netrin cues. Cell 87:187-195.

Colamarino SA, Tessier-Lavigne M (1995) The axonal chemoattractant Netrin-1 is also a chemorepellent for trochlear motor axons. Cell 81:621-629.

Colavita A, Krishna S, Zheng H, Padgett RW, Culotti JG (1998) Pioneer axon guidance by UNC-129, a C. elegans TGF-beta. Science 281:706-709.
Desai C, Garriga G, McIntire SL, Horvitz HR (1988) A genetic pathway for the development of the Caenorhabditis elegans HSN motor neurons. Nature 336:638-646.

Durbin R (1987) Studies on the development and organisation of the nervous system of Caenorhabditis elegans. PhD Thesis, University of Cambridge.

Engel J (1996) Domain organizations of modular extracellular matrix proteins and their evolution. Matrix Biol 15:295-299.

Graham PL, Johnson JJ, Wang S, Sibley MH, Gupta MC, Kramer JM (1997) Type IV collagen is detectable in most, but not all, basement membranes of Caenorhabditis elegans and assembles on tissues that do not express it. J Cell Biol 137:1171-1183.

Hall DH, Hedgecock EM (1991) Kinesin-related gene unc-104 is required for axonal transport of synaptic vesicles in C. elegans. Cell 65:837-847.

Hamelin M, Zhou Y, Su MW, Scott IM, Culotti JG (1993) Expression of the UNC-5 guidance receptor in the touch neurons of C. elegans steers their axons dorsally. Nature 364:327-330.

Harris R, Sabatelli LM, Seeger MA (1996) Guidance cues at the Drosophila CNS midline: identification and characterization of two Drosophila Netrin/UNC-6 homologs. Neuron 17:217-228.

Hart AC, Sims S, Kaplan JM (1995) Synaptic code for sensory modalities revealed by $C$. elegans GLR-1 glutamate receptor. Nature 378:82-85.

Hedgecock EM, Culotti JG, Hall DH (1990) The unc-5, unc-6, and unc-40 genes guide circumferential migrations of pioneer axons and mesodermal cells on the epidermis in C. elegans. Neuron 4:61-85.

Hekimi S, Kershaw D (1993) Axonal guidance defects in a Caenorhabditis elegans mutant reveal cell- extrinsic determinants of neuronal morphology. J Neurosci 13:4254-4271.

Ishii N, Wadsworth WG, Stern BD, Culotti JG, Hedgecock EM (1992) UNC-6, a laminin-related protein, guides cell and pioneer axon migrations in C. elegans. Neuron 9:873-881.

Keino-Masu K, Masu M, Hinck L, Leonardo ED, Chan SS-Y, Culotti JG, Tessier-Lavigne M (1996) Deleted in Colorectal Cancer (DCC) encodes a netrin receptor. Cell 87:175-185.

Kennedy TE, Serafini T, de la Torre JR, Tessier-Lavigne M (1994) Netrins are diff usible chemotropic factors for commissural axons in the embryonic spinal cord. Cell 78:425-435.

Kolodziej PA (1996) frazzled encodes a Drosophila member of the DCC immunoglobulin subfamily and is required for CNS and motor axon guidance. Cell 87:197-204.

Kramer JM, French RP, Park EC, Johnson JJ (1990) The Caenorhabditis elegans rol-6 gene, which interacts with the sqt- 1 collagen gene to determine organismal morphology, encodes a collagen. Mol Cell Biol 10:2081-2089.

Lauderdale JD, Davis NM, Kuwada JY (1997) Axon tracts correlate with netrin-1a expression in the zebrafish embryo. Mol Cell Neurosci 9:293-313.

Leonardo ED, Hinck L, Masu M, Keino-Masu K, Ackerman SL, TessierLavigne M (1997) Vertebrate homologues of C. elegans UNC-5 are candidate netrin receptors. Nature 386:833-838.

Leung-Hagesteijn C, Spence AM, Stern BD, Zhou Y, Su MW, Hedgecock EM, Culotti JG (1992) UNC-5, a transmembrane protein with immunoglobulin and thrombospondin type 1 domains, guides cell and pioneer axon migrations in C. elegans. Cell 71:289-299.

Leyns L, Bouwmeester T, Kim SH, Piccolo S, De Robertis EM (1997) Frzb-1 is a secreted antagonist of Wnt signaling expressed in the Spemann organizer. Cell 88:747-756.

Maduro M, Pilgrim D (1995) Identification and cloning of unc-119, a gene expressed in the Caenorhabditis elegans nervous system. Genetics 141:977-988.

Maricq AV, Peckol E, Driscoll M, Bargmann CI (1995) Mechanosensory signalling in $C$. elegans mediated by the GLR-1 glutamate receptor. Nature [erratum 379:749] 378:78-81.

McIntire SL, Garriga G, White J, Jacobson D, Horvitz HR (1992) Genes necessary for directed axonal elongation or fasciculation in C. elegans. Neuron 8:307-322.

Mello C, Fire A (1995) DNA Transformation. In: Caenorhabditis elegans: modern biological analysis of an organism, Vol. 48 (Epstein HF, Shakes DC, eds), pp.451-482. San Diego: Academic.

Mello CC, Kramer JM, Stinchcomb D, Ambros V (1991) Efficient gene transfer in C. elegans: extrachromosomal maintenance and integration of transforming sequences. EMBO J 10:3959-3970. 
Mitchell KJ, Doyle JL, Serafini T, Kennedy TE, Tessier-Lavigne M, Goodman CS, Dickson BJ (1996) Genetic analysis of Netrin genes in Drosophila: Netrins guide CNS commissural axons and peripheral motor axons. Neuron 17:203-215.

Otsuka AJ, Jeyaprakash A, Garcia-Anoveros J, Tang LZ, Fisk G, Hartshorne T, Franco R, Born T (1991) The C. elegans unc-104 gene encodes a putative kinesin heavy chain-like protein. Neuron 6:113-122.

Ren XC, Kim S, Fox E, Hedgecock EM, Wadsworth WG (1999) Role of netrin UNC-6 in patterning the longitudinal nerves of Caenorhabditis elegans. J Neurobiol 39:107-118.

Sambrook J, Fritsch EF, Maniatis T (1989) Molecular cloning: a laboratory manual, Ed 2. Cold Spring Harbor, NY: Cold Spring Harbor Laboratory.

Serafini T, Kennedy TE, Galko MJ, Mirzayan C, Jessell TM, TessierLavigne M (1994) The netrins define a family of axon outgrowthpromoting proteins homologous to C. elegans UNC-6. Cell 78:409-424.

Shirasaki R, Katsumata R, Murakami F (1998) Change in chemoattractant responsiveness of developing axons at an intermediate target. Science 279:105-107.

Spivak-Kroizman T, Lemmon MA, Dikic I, Ladbury JE, Pinchasi D, Huang J, Jaye M, Crumley G, Schlessinger J, Lax I (1994) Heparininduced oligomerization of FGF molecules is responsible for FGF receptor dimerization, activation, and cell proliferation. Cell 79:1015-1024.

Sternberg PW, Horvitz HR (1986) Pattern formation during vulval development in C. elegans. Cell 44:761-772.

Strahle U, Fischer N, Blader P (1997) Expression and regulation of a netrin homologue in the zebrafish embryo. Mech Dev 62:147-160.

Sulston J, Hodgkin J (1988) Methods. In: The nematode Caenorhabditis elegans (Wood WB, ed), pp 587-606. Cold Spring Harbor, NY: Cold Spring Harbor Laboratory.

Sulston J, Schierenberg E, White J, Thomson J (1983) The embryonic cell lineage of the nematode Caenorhabditis elegans. Dev Biol 100:64-119.

Tessier-Lavigne M, Goodman CS (1996) The molecular biology of axon guidance. Science 274:1123-1133.

Wadsworth WG, Hedgecock EM (1992) Guidance of neuroblast migrations and axonal projections in Caenorhabditis elegans. Curr Opin Neurobiol 2:36-41.

Wadsworth WG, Hedgecock EM (1996) Hierarchical guidance cues in the developing nervous system of C. elegans. Bioessays 18:355-362.

Wadsworth WG, Bhatt H, Hedgecock EM (1996) Neuroglia and pioneer neurons express UNC-6 to provide global and local netrin cues for guiding migrations in C. elegans. Neuron 16:35-46.

Walz A, McFarlane S, Brickman YG, Nurcombe V, Bartlett PF, Holt CE (1997) Essential role of heparan sulfates in axon navigation and targeting in the developing visual system. Development 124:2421-2430.

Wang S, Krinks M, Lin K, Luyten FP, Moos Jr M (1997) Frzb, a secreted protein expressed in the Spemann organizer, binds and inhibits Wnt-8. Cell 88:757-766.

Wightman B, Clark SG, Taskar AM, Forrester WC, Maricq AV, Bargmann CI, Garriga G (1996) The C. elegans gene vab-8 guides posteriorly directed axon outgrowth and cell migration. Development 122:671-682.

Wolf FW, Hung MS, Wightman B, Way J, Garriga G (1998) vab-8 is a key regulator of posteriorly directed migrations in C. elegans and encodes a novel protein with kinesin motor similarity. Neuron 20: $655-666$. 